\title{
Angiographic Review of Choroidal Involution in Eyes with Retinopathy of Prematurity Treated with Bevacizumab
}

\author{
Yasmin Islam Lorick Andersen Swati Agarwal-Sinha \\ Department of Ophthalmology, University of Florida College of Medicine, Gainesville, FL, USA
}

\section{Keywords}

Anti-vascular endothelial growth factor · Retinopathy of prematurity · Choroidal involution · Fluorescein angiography

\begin{abstract}
Purpose: Retinopathy of prematurity (ROP) is considered a disease of the inner retina; however, there is increasing evidence that demonstrates choroidal vasculature loss in ROP, leading to degeneration of outer retinal function and visual deterioration. Central choroidal thinning is noted in children with history of ROP using optical coherence tomography imaging. This study characterizes the presence and persistence of choroidal loss angiographically in eyes of infants treated with intravitreal bevacizumab (IVB) for stage 3 ROP. Methods: The fluorescein angiography (FA) images of 62 eyes of 31 infants treated with IVB monotherapy were retrospectively reviewed. The eyes with good quality early-, mid-, and latephase imaging were included in this study. The presence of choroidal hypofluoresence involving the central and or peripheral retina was noted. In infants with multiple FAs, serial FAs were analyzed for persistence of choroidal hypofluorescence. Results: The mean age and birth weight of infants was 24.4 weeks post-menstrual age and $683 \mathrm{~g}$, respectively. All infants received IVB monotherapy. Twenty-four of 62 angi-
\end{abstract}

ography images of sufficient quality reviewed showed the presence of choroidal hypofluorescence involving central and peripheral lobular loss in the early phase and its persistence into mid-and late phases. Twelve eyes demonstrated persistent choroidal loss on sequential FA until 3 years chronological age. Conclusions: The study demonstrates the presence of choroidal vascular loss angiographically both central and peripheral fundus in infants with ROP. It highlights the critical role of choroidal involution in outer retinal function that could affect visual outcomes.

(c) 2020 S. Karger AG, Base

\section{Introduction}

Retinopathy of prematurity (ROP) is the leading cause of pediatric vision loss in high-income countries, and it is increasingly a cause of vision loss in middle- and lowincome countries [1]. The pathogenesis of ROP involves exposure to higher levels of oxygen after premature birth than in utero. The initial phase of ROP involves cessation of normal retinal vasculature growth and regression of the retinal vasculature, followed by a second phase of hypoxia-induced retinal neovascularization. As such, vision loss in ROP has traditionally been described as a result of pathology in the inner retinal vasculature [2].
Correspondence to:

Swati Agarwal-Sinha, sxapublish@gmail.com 
Abnormalities in the inner retinal vasculature are typically seen in the peripheral retina, with relative sparing of the central retina [2]. However, prior studies using multifocal electroretinogram have demonstrated that the majority of functional vision loss in ROP is due to central retinal dysfunction [3]. Notably, cone dysfunction and foveal dysplasia have been described; these do not correlate to the relative sparing of retinal vasculature abnormalities in the macula [4-6]. As a result of these findings, recent research has begun to focus on the choroidal vasculature as a possible etiology of vision loss in patients with ROP. Oxygen-induced retinopathy (OIR) models in rat models have demonstrated that choroidal vasculature involutes in ROP, indicating a possible mechanism for central vision loss in these patients [7].

Optical coherence tomography (OCT) imaging in infants with ROP demonstrates choroidal thinning when compared to infants without ROP, suggesting that choroidal vasculopathy translates to infants with ROP [810]. Choroidal involution and thinning persists in patients with regressed ROP, thus indicating that these changes are permanent and a cause of long-term vision loss in these patients [11]. OCT studies performed to date document the central choroidal thickness $[8,9,11]$ but have not studied the choroidal vasculature directly or peripheral choroidal loss due to limitations of this technology in studying vasculature itself. Lavric et al. [12] used swept-source OCT to study the choroidal vascularity index and found that in children ages $5-15$ years with a history of ROP, the choroidal vascularity index was significantly reduced. However, they did not study the choroidal vasculature directly using angiography techniques [12]. Unlike OCT, fluorescein angiography (FA) can be performed in premature infants, providing an opportunity to characterize associated choroidal vascular loss along with inner retinal abnormalities in this patient population.

The status of the choroidal vasculature may also vary with the treatment used to combat ROP, as the normal development of the choroidal vasculature is dependent on vascular endothelial growth factor (VEGF) [13]. Since the advent of the BEAT-ROP study in 2011, intravitreal bevacizumab (IVB), an anti-VEGF agent, has been increasingly used to treat patients with ROP [14]. It avoids the permanent destruction of the retina associated with panretinal photocoagulation or cryotherapy. However, treatment with IVB has been demonstrated to have late recurrences of stage 4 and 5 ROP beyond until 3 years of age [15-17], thus requiring frequent retinal examinations. FA is a relatively safe procedure commonly used by the providers to identify and document recurrent ROP and is used to study the retinal vasculature after treatment with bevacizumab [18-20]. However, the literature on the use of FA in patients with ROP focuses on the retinal vasculature with little mention of the choroidal circulation. The purpose of this study was to angiographically document the presence of choroidal vasculature loss in infants with stage $3 \mathrm{ROP}$ and to analyze if these changes persist over time.

\section{Methods}

This retrospective, observational study included premature infants undergoing screening and treatment for ROP at an academic center between 2014 and 2019 (University of Florida, Gainesville, FL, USA). Screening for ROP was conducted for all infants born at $<31$ weeks gestational age (GA), <1,501 $\mathrm{g}$ birth weight (BW), or those who had an unstable clinical course as judged by their neonatologist. Classification of ROP was conducted based on the International Classification for Retinopathy of Prematurity [21]. This study was approved by the Institutional Review Board at the University of Florida and was compliant with the Health Insurance Portability and Accountability Act. Informed consent was obtained from all parents and/or legal guardians of the infants participating in the study.

\section{Treatment and Monitoring for ROP Recurrence}

In our practice, infants receiving IVB monotherapy are treated with $0.75 \mathrm{mg} / 0.03 \mathrm{~mL}$ IVB. Afterward, they receive eye examinations weekly until 55 weeks of post-menstrual age (PMA). The exams are then extended to exams every $4-5$ weeks. Around $65-70$ weeks PMA, an examination under anesthesia with FA is performed in the operating room.

Under anesthesia, depressed retinal examination using binocular indirect ophthalmoscopy is performed to assess the stage and zone of ROP of any recurrence of sight-threatening stages of ROP. The same dose of bevacizumab is used for treating recurrent stage $3 \mathrm{ROP}$, if present. Digital FA is performed using a bolus of $10 \%$ fluorescein sodium administered intravenously at a dose of $0.1 \mathrm{~mL} / \mathrm{kg}$, followed by a saline flush. In each FA, the first eye in which imaging was taken was considered the transit eye, which in the majority of patients was the right eye. All infants had fundus and FA imaging performed using the RetCam imaging system (Clarity Medical Systems, Pleasanton, CA, USA). Sequential FAs and examinations under anesthesia are performed every 6-8 months during the first 3 years of life to monitor for recurrence of ROP and to evaluate avascular peripheral retinal vasculature in these infants.

While there is no clearly defined standard of care for the frequency of examinations in infants previously treated with bevacizumab for ROP beyond 65 weeks PMA [22], we pursue this aggressive approach to ensure that recurrence of ROP is not missed with possibly visually devastating outcomes due to multiple reports of recurrence up to 3 years of age [15-17]. Peripheral retinal examinations in clinic are limited in children due to cooperation. In one prior case report, a child developed reactivation of ROP and developed bilateral tractional retinal detachments at 3 years of age after 

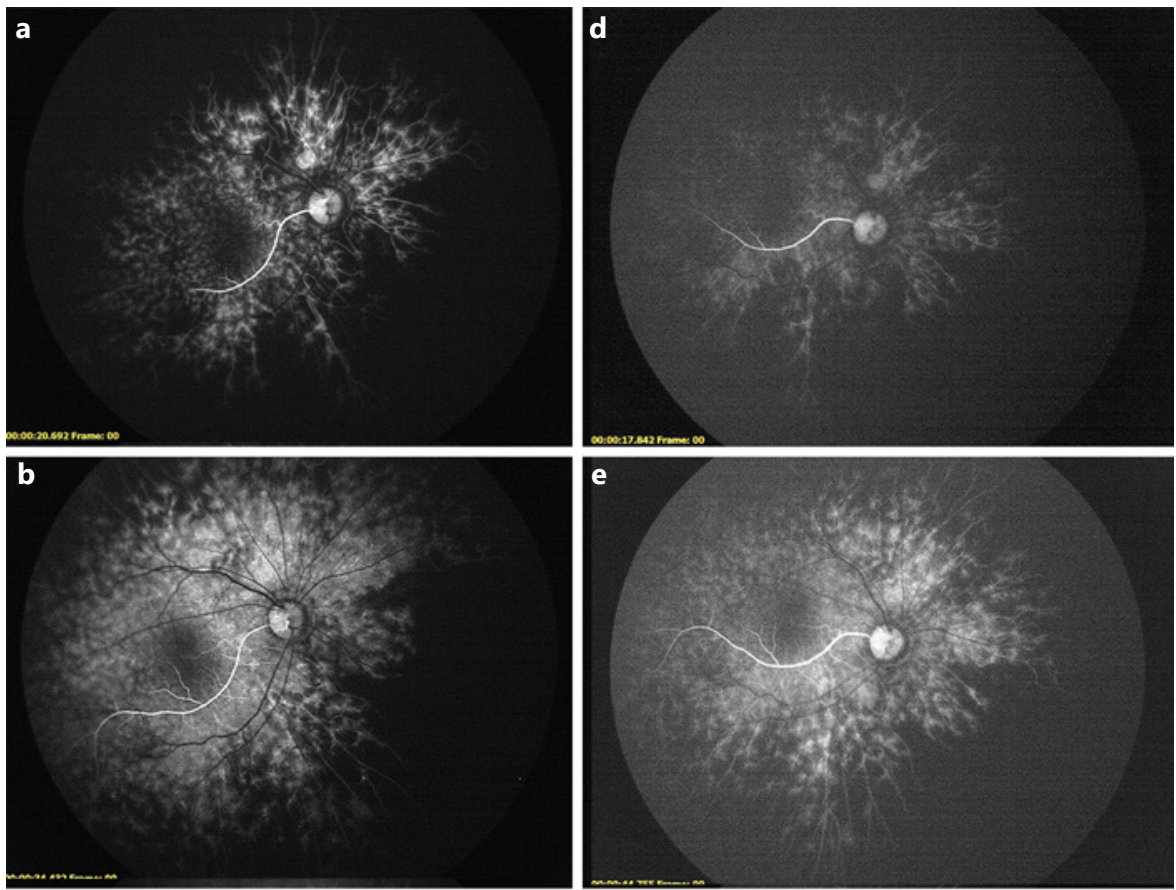

Fig. 1. Male infant born at $233 / 7$ weeks GA both eyes for zone 1 , stage 3 with plus disease at 35 weeks PMA. a-c Angiograms of the right eye at 73 5/7 weeks PMA. a The early choroidal filling phase, demonstrating patchy choroidal filling centrally with large areas of no choroidal filling and in the nasal and inferior periphery beyond the arcades. These findings persist in the mid (b) and late phase (c). d-f At 130 5/7 weeks (2 years of chronological age), a similar choroidal involution filling pattern is seen in the same eye.
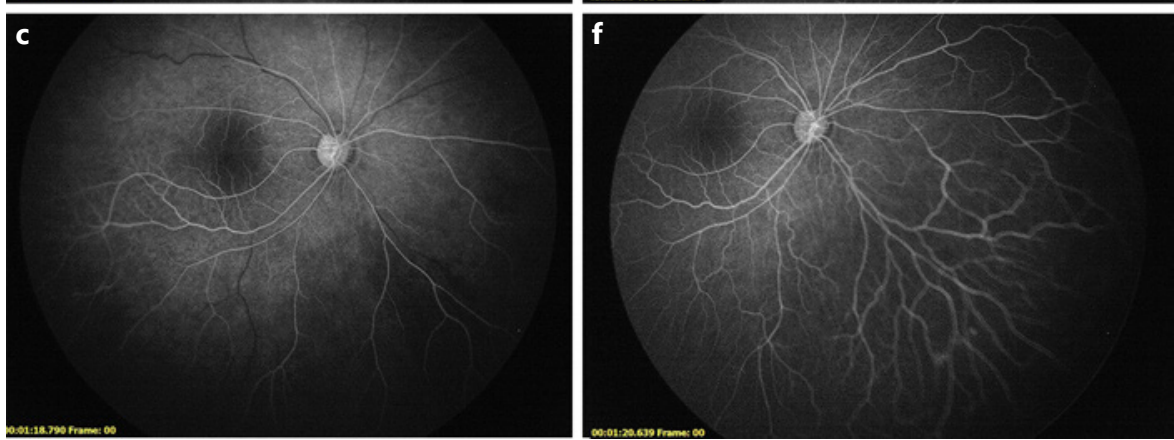

receiving examinations in clinic every 6 months [15]. Thus, by performing frequent examinations under anesthesia, we aim to identify recurrences early to prevent stage 4 and 5 ROP from developing. A total of 31 infants (62 eyes) treated with IVB monotherapy received at least one FA as part of monitoring the progression of ROP and are included in this study.

\section{Data Collection}

The images were independently reviewed by two physicians. The angiography imaging of the eye which had good quality early-, mid-, and late-phase images were included in the study. Some patients underwent more than one FA; in these cases, all subsequent FAs were reviewed. Serial images were evaluated for each eye throughout the course of each FA. Images involving central loss and or peripheral lobular loss in early choroidal phase and midand late phases on FA were considered to indicate choroidal involution. The presence and extent of choroidal hypofluorescence involving the central and or peripheral retina were noted. A chart review was then performed to determine each patient's GA at birth, age at which they received IVB, BW, and age at which they received a diagnosis of threshold ROP.

Angiographic Review of Choroidal Involution in Anti-VEGF-Treated ROP
Table 1. Demographics of infants included in the study

\begin{tabular}{ll}
\hline Variable & Patients, $n$ \\
\hline Sex & 18 males; 13 females \\
Average GA at birth & 24.5 weeks PMA \\
Average BW & $668 \mathrm{~g}$ \\
Average GA at diagnosis of type 1 ROP & 33.6 weeks PMA \\
Average GA at IVB treatment & 36.4 weeks PMA \\
Average GA at first FA & 67.2 weeks PMA
\end{tabular}

\section{Results}

Thirty-one infants (62 eyes) treated with IVB monotherapy for type 1 ROP were included in the study. Their demographics are included in Table 1. The mean age at birth was 24.4 weeks GA and average BW was 668 grams. The infants received first IVB at an average of 36.4 weeks 

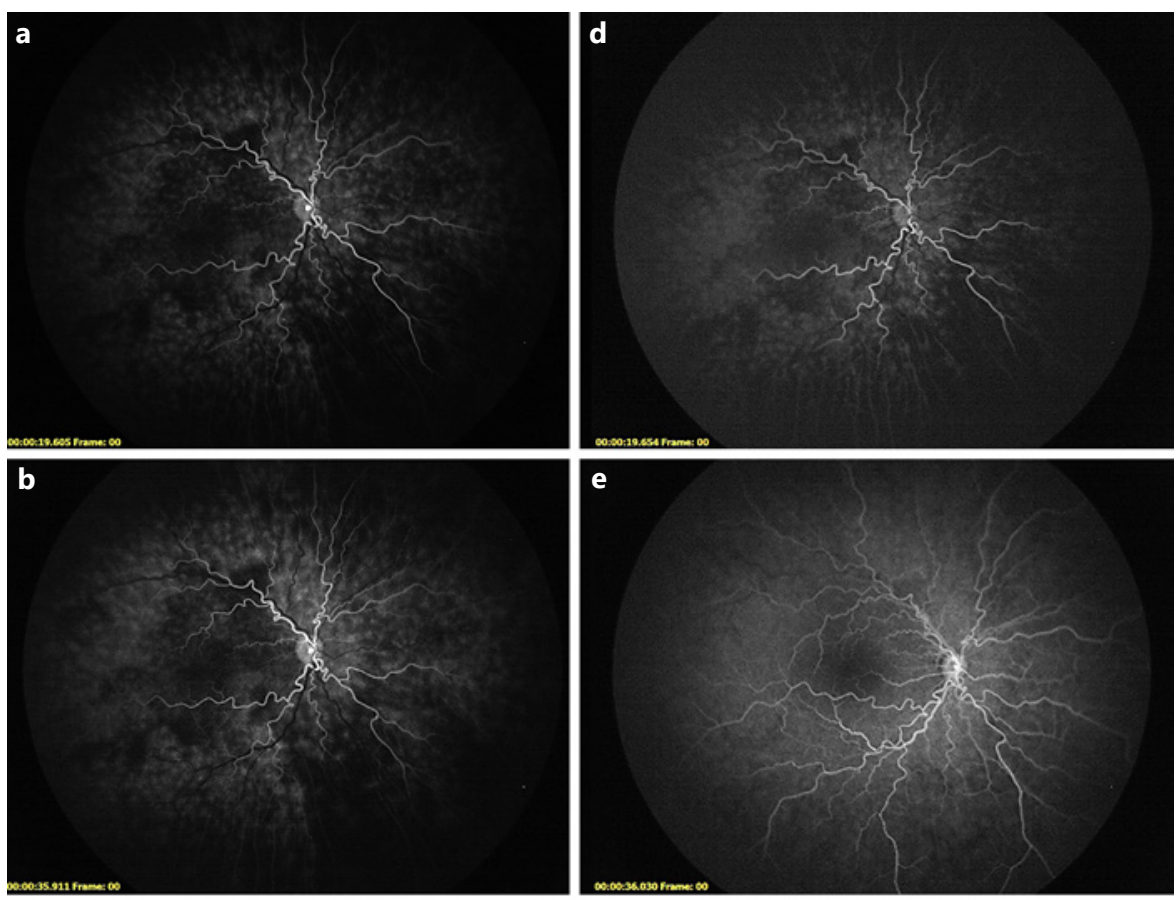

Fig. 2. Male infant born at 23 weeks GA with $710 \mathrm{~g} \mathrm{BW}$. Treated with IVB in both eyes at 31 6/7 weeks PMA for zone 1, stage 3 with plus disease. a-c Angiograms of the right eye at 107 2/7 weeks PMA (19 months chronological age). The early choroidal filling phase (a) demonstrates patchy choroidal filling centrally with large areas of no choroidal filling and in the nasal periphery beyond the arcades persistent in the mid (b) and late phase (c).d-f At 153 2/7 weeks ( $2 \frac{1}{2}$ years of chronological age), the similar choroidal involution filling pattern is seen.
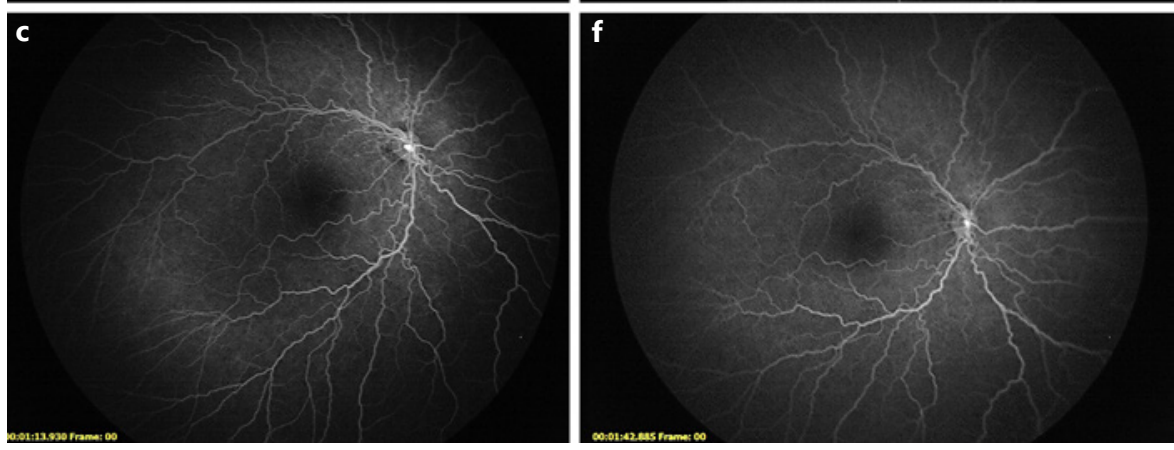

PMA. The mean GA at first FA was 67.2 weeks (range 44-82 weeks PMA). Twenty infants received more than one FA during the study period.

Twenty-four of the 62 eyes included in the study had angiography images that were of sufficient quality to evaluate choroidal perfusion during early-, middle-, and late phases. The high number of eyes with insufficient quality images was typically due to no imaging of the early phase, as it was the non-transit eye (e.g., the right eye was imaged first, and thus the left eye did not receive early choroidal filling images) or poor image quality.

The normal choroidal filling pattern expected was complete filling of the choriocapillaris by the early arterial phase. These FA images taken in our study demonstrated choroidal hypofluorescence involving central retina, peripheral lobular loss, or both (Fig. 1-3). Choroidal hypofluorescence was noted in the early choroidal phase and persisted in the mid- and late phases on
FA. Twelve of the 24 eyes with good quality images had sequential FAs as late as 3 years of chronological age; all of these eyes demonstrated persistent choroidal hypofluorescence in a similar pattern at all different time points.

\section{Discussion}

ROP has been characterized for decades as abnormal retinal vascularization, and subsequently, it has been classified into stages based on the retinal vasculature. However, newer studies have shown the choroidal vasculature is also compromised in ROP; thus, ROP is both a disease of the inner and outer retina. The outer retinal abnormalities result in degeneration of the retinal pigment epithelium (RPE) and the photoreceptors, causing permanent visual dysfunction $[8,9,11,23]$. 

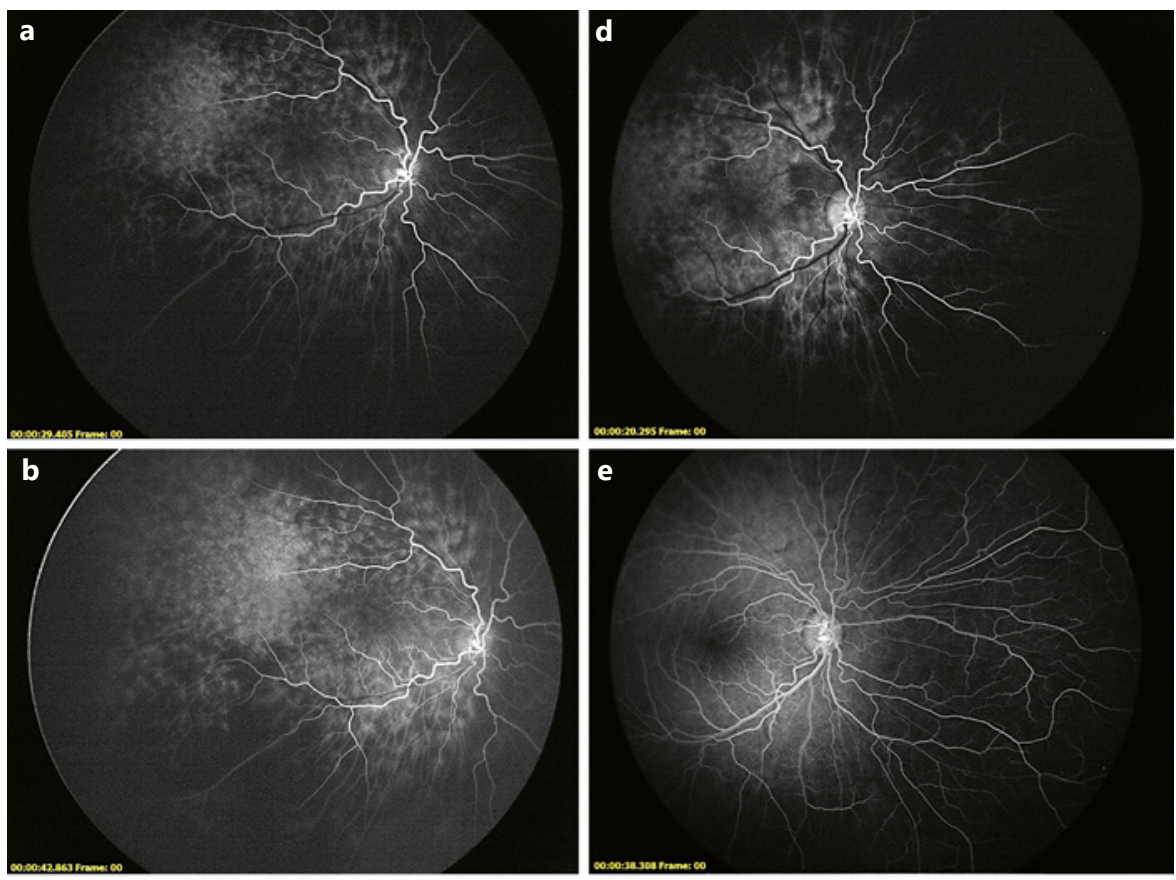

Fig. 3. Female infant born at 24 weeks GA with BW of $615 \mathrm{~g}$. Treated with IVB in the right eye at 39 weeks PMA for zone 1, stage 3 with plus. a-c Angiograms of the right eye at $1506 / 7$ weeks PMA (2 years of chronological age). a The early phase of the angiogram, which demonstrates patchy choroidal filling centrally with large areas of no choroidal filling and in the nasal, inferior, and superior periphery beyond the arcades persistent in the mid (b) and late phase (c). d-f At 187 6/7 weeks (3 years of chronological age), a similar choroidal filling pattern is seen, demonstrating persistent choroidal involution.
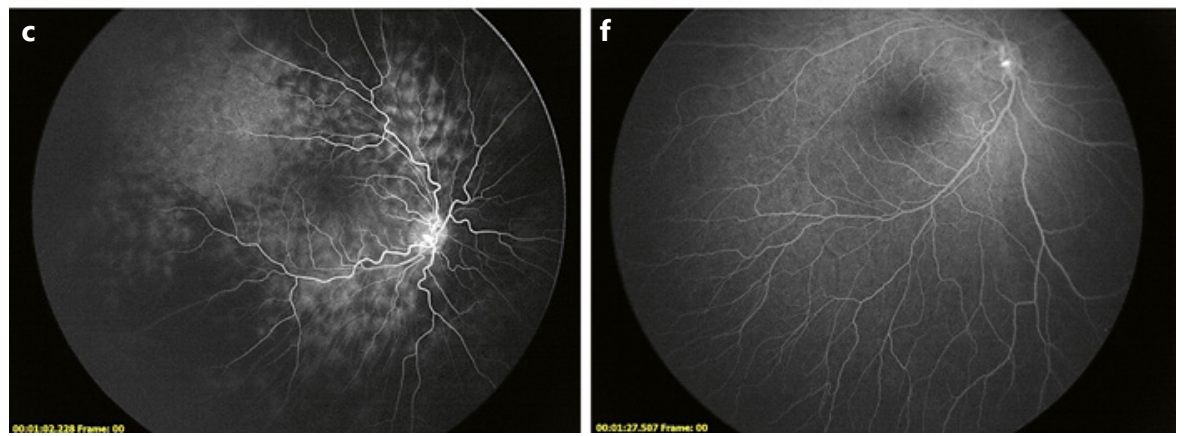

The choroidal vasculature originates from the ophthalmic artery as short posterior ciliary arteries. These supply the posterior choroid, while the long posterior ciliary arteries vascularize the anterior choroid, iris, and the ciliary body. The choroidal vasculature is the exclusive source of nutrients and oxygen to the outer retina, including the RPE and photoreceptors. The inner retinal layers are supplied by the vascular network arising from the central retinal artery, which itself is a branch of the ophthalmic artery [24].

Embryologically, mesoderm begins to differentiate into endothelial cells at fourth week of gestation, forming the precursors of the choriocapillaris. The choroidal capillary network becomes organized by eighth week of gestation, and it branches extensively by eleventh week of gestation. The choroidal vasculature subsequently matures by the third and fourth months of gestation. In contrast, the retina remains avascular until the fourth month of gestation; prior to this, the hyaloid vasculature provides nutrition to the developing retina. In the fourth month of gestation, the hyaloid vasculature begins to regress as the first retinal vessels appear from the optic nerve head, forming a primitive central retinal arterial system. At 6 months of gestation, the retinal vessels begin extending and migrating outwards towards the ora serrata, reaching their destination by 36 weeks of gestation nasally and 40 weeks of gestation temporally. Thus, infants born at term have a fully vascularized retina at birth $[13,25]$.

The pathogenesis of ROP is multifactorial. Besides prematurity, low BW and supplemental oxygen demand have been implicated as risk factors in the development and progression of ROP [26-28]. Chemical mediators, including VEGF, placental growth factor (PlGF), erythropoietin, insulin-like growth factor (IGF-1), and others also hold an important role in angiogenesis in this dis- 
ease. VEGF-A and PIGF have been implicated to have a potential role in retinal neovascularization [29-32]. In recent studies, intrauterine inflammation, chronic placental inflammation, oxidative stress, and generalized inflammation are noted as major risk factors in the pathological angiogenesis, development, and progression of ROP in both human and animal studies [33-38]. Additionally, inflammatory mediators, such as IL-6, IL-18, cyclooxygenase- 2 , and IL- $1 ß$, are elevated in OIR models of ROP. IL- $1 ß$ has been implicated as one of the main cytokines involved in the retinal and neuronal vascular degeneration in ischemic retinopathies. IL- $1 ß$ is shown to play a critical role in choroidal degeneration and outer retinal dysfunction. Recombinant IL- $1 ß$ receptor antagonist use in OIR models have shown to protect against laser-induced choroidal neovascularization, neuronal damage, and vascular degeneration, thus protracting outer retina and visual function $[39,40]$.

Prior literature has also described pathology of the choroid in patients with ROP. Choroidal involution in prematurity has been studied in children using spectral domain optical coherence tomography imaging. This imaging has demonstrated central choroidal thinning in premature infants $[8,9]$, and these findings extend to young adulthood $[11,12]$. However, prior studies have not correlated this with angiographic study of the choroidal vasculature. In this study, we documented the existence of choroidal loss at an average of 67 weeks PMA by FA imaging in premature infants with type 1 ROP treated with IVB monotherapy. This study also highlights the involvement of peripheral lobular choroidal loss extending beyond the central retina in infants with ROP. As all of the infants in our study were treated with IVB, we are unable to definitively determine if the choroidal involution is due to treatment with bevacizumab or the underlying ROP. However, it is unlikely that bevacizumab caused the choroidal involution seen in this study, as the choroidal vessels undergo involution due to hypoxia in phase 1 of ROP, whereas bevacizumab is not given until phase 2, when retinal neovascularization has developed. Besides, the choroidal vasculature embryologically matures by 3-4 months of gestation. Additionally, other studies have reported choroidal thinning in eyes with ROP not treated with bevacizumab, further supporting that hypoxia and other inflammatory mediators like IL- $1 ß$ plays a critical role in choroidal involution documented angiographically in our study $[8,9,11,39,40]$.

One of the limitations of this study was multiple FAs did not include early-phase images of sufficient quality to be able to reliably evaluate the choroidal flush and subse- quently track areas of choroidal hypofluorescence through the remainder of the angiogram. Second, the study does not include visual function to correlate with the choroidal loss, as it was not possible to obtain reliable visual function testing at the ages included in this study ( 3 years of age and younger). We aim to describe visual function in a follow-up study. Additionally, due to the risks of administering general anesthesia and the risks of FA, which include anaphylaxis, no healthy control infants without ROP were included in this study.

One challenge of performing FA in infants is the mechanism by which images must be captured; unlike in cooperative adults, in which photos can be taken while the patient fixates on a target by a non-contact camera, the RetCam being a contact camera applies pressure to the eye, which may result in transient delay of choroidal and retinal perfusion due to raised intraocular pressure (IOP), thus confounding results [41]. In a study done in the 2 primate monkeys, the authors raised the IOP to 35 and $60 \mathrm{~mm}$ of $\mathrm{Hg}$ and then performed the FA and indocyanine green (ICG) angiography. The study showed no perfusion delays in the choriocapillaries at $35 \mathrm{~mm} \mathrm{Hg}$ with FA and ICG [41]. To decrease this confounding, all images were taken by a single experienced pediatric ophthalmologist (SAS) who was cognizant of the changes that could potentially be induced by raising IOP, and she thus attempted to put as minimal pressure as possible on the eye when obtaining the images. We are confident the choroidal involution changes seen are not from raised IOP as first the angiography imaging was performed under general anesthesia, which was helpful in negating the head and eye movement of the infants. Second, the transient raise in IOP would be unlikely to provide the consistent findings of choroidal involution in all early-, mid-, and late angiography phases in the same imaging session, as well as overtime as the child ages, as it is unlikely that the same pressure was applied at each time point. Third, OCT imaging studies has documented central choroidal thinning in older children with ROP, emphasizing that the choroidal vasculature does undergo involution in the early phase of ROP in premature infants.

The timing of the FA (early, mid, or late phase) was unable to be judged based on the time since fluorescein bolus, as sometimes in children, intravenous access is obtained via either the foot or the hand, thus altering the limb-to-eye time. Thus, the timing of each image was based on the filling of the vasculature. Additionally, under normal physiologic conditions, the temporal choroid may have delayed filling as compared to the remainder of the choroid, as this area is supplied by retrograde choroidal arteries [42]. Thus, it is more chal- 
lenging to assess for delayed choroidal filling in the temporal retina.

ICG angiography would be an ideal choice to study the choroidal vasculature; however, ICG requires infrared video angiography that is currently not available for pediatric imaging. However, FA can be performed bedside and or in a standard operating room with equipment already possessed by most centers who evaluate and treat ROP. In fact, FA has been demonstrated as a valuable tool in studying retinal vasculature in infants with ROP [20, 43]. By obtaining early-phase images demonstrating choroidal flush and following the choroidal pattern in these areas throughout the remainder of the angiogram, this study demonstrates that FA can appropriately be used to evaluate the choroidal vasculature in infants with ROP.

In conclusion, our study demonstrates choroidal vascular loss on angiography in infants with type $1 \mathrm{ROP}$ treated with IVB monotherapy. Abnormalities in the choroidal vasculature were seen involving both the central posterior pole and peripheral fundus. This choroidal vasculopathy was seen up to 3 years of age, indicating that these changes may be permanent. This decrease in choroidal vasculature can help explain the central retinal dysfunction and photoreceptor malfunction seen in patients with ROP, thereby providing a mechanism by which patients with ROP can have visual impairment unexplained solely by the retinal vascular pathology. Further studies are indicated to study the choroidal vasculature into later childhood and adulthood, and to also specifically address the effect of choroidal involution on photoreceptor and RPE function in infants with ROP.

\section{Acknowledgements}

We thank the OR staff at the University of Florida, Jock, and Sharon for the assistance provided in performing the fluorescein angiography in infants.

\section{Statement of Ethics}

The study protocol was approved by the institute's committee on human or animal research (IRB201702677). Written informed consent was obtained from all parents and/or legal guardians of the infants participating in the study.

\section{Conflict of Interest Statement}

The authors have no conflicts of interest to declare.

\section{Funding Sources}

This study received no financial support for the research, authorship, and or publication of this article.

\section{Author Contributions}

Yasmin Islam, MD (PGY-4), contributed to literature search, figures, data analysis, original draft preparation, editing, and revising. Lorick Andersen, MD, contributed to literature search, figures, data analysis, editing, and revising. Swati Agarwal-Sinha, $\mathrm{MD}$, operated the RetCam $\odot$ and took fundus photographs, contributed to figures, study design, data collection, data analysis, editing, writing, and final approval of the manuscript.

\section{Data Availability Statement}

The data that support the findings of this study are not publicly available due to their containing information that could compromise the privacy of patient but are available from the corresponding author (SAS) upon reasonable request.

\section{Meeting Presentations}

1. Annual Virtual Meeting of the Association for Research in Vision and Ophthalmology 2020, Baltimore, MD, USA.

2. Annual Virtual Meeting of the American Society of Retina Specialists 2020, Seattle, WA, USA.

\section{References}

1 Gilbert C, Foster A. Childhood blindness in the context of VISION 2020: the right to sight. Bull World Health Organ. 2001;79:227-32.

2 Chen J, Smith LE. Retinopathy of prematurity. Angiogenesis. 2007;10:133-40.

3 Siatkowski RM, Dobson V, Quinn GE, Summers CG, Palmer EA, Tung B. Severe visual impairment in children with mild or moderate retinal residua following regressed threshold retinopathy of prematurity. J AAPOS. 2007;11:148-52.

Angiographic Review of Choroidal Involution in Anti-VEGF-Treated ROP
4 Recchia FM, Recchia CC. Foveal dysplasia evident by optical coherence tomography in patients with a history of retinopathy of prematurity. Retina. 2007;27:1221-6

5 Soong GP, Shapiro M, Seiple W, Szlyk JP. Macular structure and vision of patients with macular heterotopia secondary to retinopathy of prematurity. Retina. 2008;28:1111-6.

6 Baker PS, Tasman W. Optical coherence tomography imaging of the fovea in retinopathy of prematurity. Ophthalmic Surg Lasers Imaging. 2010;41:201-6.
7 Shao Z, Dorfman AL, Seshadri S, Djavari M, Kermorvant-Duchemin E, Sennlaub F, et al. Choroidal involution is a key component of oxygen-induced retinopathy. Invest Ophthalmol Vis Sci. 2011;52:6238-48.

8 Moreno TA, O'Connell RV, Chiu SJ, Farsiu S, Cabrera MT, Maldonado RS, et al. Choroid development and feasibility of choroidal imaging in the preterm and term infants utilizing SD-OCT. Invest Ophthalmol Vis Sci. 2013;54:4140-7. 
9 Erol MK, Coban DT, Ozdemir O, Dogan B, Tunay ZO, Bulut M. Choroidal thickness in infants with retinopathy of prematurity. Retina. 2016;36:1191-8.

10 Ulusoy MO, Kıvanç SA, Kal A. Evaluation of iridocorneal angle, choroidal thickness and retinal nerve fiber kayer thickness in children with a history of retinopathy of prematurity. J Glaucoma. 2020;29(2):112-6.

11 Anderson MF, Ramasamy B, Lythgoe DT, Clark D. Choroidal thickness in regressed retinopathy of prematurity. Eye. 2014;28:14618.

12 Lavric A, Tekavcic Pompe M, Markelj S, Ding J, Mahajan S, Khandelwal N, et al. Choroidal structural changes in preterm children with and without retinopathy of prematurity. Acta Ophthalmol. 2019 Dec. Epub ahead of print.

13 Anand-Apte B, Hollyfield J. Developmental anatomy of the retinal and choroidal vasculature. Elsevier; 2010.

14 Mintz-Hittner HA, Kennedy KA, Chuang AZ; BEAT-ROP Cooperative Group. Efficacy of intravitreal bevacizumab for stage $3+$ retinopathy of prematurity. N Engl J Med. 2011; 364:603-15.

15 Agarwal-Sinha S, Guevara JG, Amin SM. Late-onset tractional fibrovascular proliferation post-intravitreal bevacizumab following treatment of retinopathy of prematurity. Can J Ophthalmol. 2018;53:e99-103.

16 Hajrasouliha AR, Garcia-Gonzales JM, Shapiro MJ, Yoon H, Blair MP. Reactivation of retinopathy of prematurity three years after treatment with bevacizumab. Ophthalmic Surg Lasers Imaging Retina. 2017;48:255-9.

$17 \mathrm{Hu}$ J, Blair MP, Shapiro MJ, Lichtenstein SJ, Galasso JM, Kapur R. Reactivation of retinopathy of prematurity after bevacizumab injection. Arch Ophthalmol. 2012;130:1000-6.

18 Zepeda-Romero LC, Oregon-Miranda AA, Lizarraga-Barrón DS, Gutiérrez-Camarena O, Meza-Anguiano A, Gutiérrez-Padilla JA. Early retinopathy of prematurity findings identified with fluorescein angiography. Graefes Arch Clin Exp Ophthalmol. 2013; 251:2093-7.

19 Park SW, Jung HH, Heo H. Fluorescein angiography of aggressive posterior retinopathy of prematurity treated with intravitreal antiVEGF in large preterm babies. Acta Ophthalmol. 2014;92:810-3.

20 Mansukhani SA, Hutchinson AK, Neustein R, Schertzer J, Allen JC, Hubbard GB. Fluorescein angiography in retinopathy of prematurity: comparison of infants treated with bevacizumab to those with spontaneous regression. Ophthalmol Retina. 2019;3:436-43.
21 International Committee for the Classification of Retinopathy of Prematurity. The international classification of retinopathy of prematurity revisited. Arch Ophthalmol. 2005; 123:991-9.

22 Fierson WM; American Academy of Pediatrics Section on Ophthalmology; American Academy of Ophthalmology; American Association for Pediatric Ophthalmology and Strabismus; American Association of Certified Orthoptists. Screening examination of premature infants for retinopathy of prematurity. Pediatrics. 2018;142(6):e20183061.

23 Wu WC, Shih CP, Wang NK, Lien R, Chen YP, Chao AN, et al. Choroidal thickness in patients with a history of retinopathy of prematurity. JAMA Ophthalmol. 2013;131: 1451-8.

24 Yu DY, Cringle SJ, Alder V, Su EN. Intraretinal oxygen distribution in the rat with graded systemic hyperoxia and hypercapnia. Invest Ophthalmol Vis Sci. 1999;40:2082-7.

25 Saint-Geniez M, D’Amore PA. Development and pathology of the hyaloid, choroidal and retinal vasculature. Int J Dev Biol. 2004;48: 1045-58.

26 Patz A. The effect of oxygen on immature retinal vessels. Invest Ophthalmol. 1965;4:98899.

27 McColm JR, Cunningham S, Wade J, Sedowofia K, Gellen B, Sharma T, et al. Hypoxic oxygen fluctuations produce less severe retinopathy thanhyperoxic fluctuations in a rat model of retinopathy of prematurity. Pediatr Res. 2004;55:107-13.

28 The STOP-ROP Multicenter Study Group. Supplemental therapeutic oxygen for prethreshold retinopathy of prematurity (STOP$\mathrm{ROP}$ ), a randomized, controlled trial. I: primary outcomes. Pediatrics. 2000;105:295310.

29 Smith LE, Kopchick JJ, Chen W, Knapp J, Kinose F, Dalley D, et al. Essential role of growth hormone in ischemia-induced retinal neovascularization. Science. 1997;276:1706-9.

30 Smith LE, Shen W, Perruzzi C, Soker S, Kinose $\mathrm{F}, \mathrm{Xu} \mathrm{X}$, et al. Regulation of vascular endothelial growth factor-dependent retinal neovascularization by insulin-like growth factor-1 receptor. Nat Med. 1999;5:1390-5.

31 Zhou AY, Bai YJ, Zhao M, Yu WZ, Huang LZ, Li XX. Placental growth factor expression is reversed by antivascular endothelial growth factor therapy under hypoxic conditions. World J Pediatr. 2014;10:262-70.

32 Morita M, Ohneda O, Yamashita T, Takahashi S, Suzuki N, Nakajima O, et al. HLF/ HIF-2alpha is a key factor in retinopathy of prematurity in association with erythropoietin. EMBO J. 2003;22:1134-46.

33 Pietrasanta C, Pugni L, Merlo D, Acaia B, Consonni D, Ronchi A, et al. Impact of different stages of intrauterine inflammation on outcome of preterm neonates: gestational age-dependent and -independent effect. PLoS One. 2019;14:e0211484.

34 Aouiss A, Anka Idrissi D, Kabine M, Zaid Y. Update of inflammatory proliferative retinopathy: ischemia, hypoxia and angiogenesis. Curr Res Transl Med. 2019;67:62-71.

35 Kim CY, Jung E, Kim EN, Kim CJ, Lee JY, Hwang JH, et al. Chronic placental inflammation as a risk factor of severe retinopathy of prematurity. J Pathol Transl Med. 2018;52: 290-7.

36 Lynch AM, Berning AA, Thevarajah TS, Wagner BD, Post MD, McCourt EA, et al. The role of the maternal and fetal inflammatory response in retinopathy of prematurity. Am J Reprod Immunol. 2018;80:e12986.

37 Rivera JC, Dabouz R, Noueihed B, Omri S, Tahiri H, Chemtob S. Ischemic retinopathies: oxidative stress and inflammation. Oxid Med Cell Longev. 2017;2017:3940241.

38 Rivera JC, Holm M, Austeng D, Morken TS, Zhou TE, Beaudry-Richard A, et al. Retinopathy of prematurity: inflammation, choroidal degeneration, and novel promising therapeutic strategies. J Neuroinflammation. 2017;14 165.

39 Olson JL, Courtney RJ, Rouhani B, Mandava $\mathrm{N}$, Dinarello CA. Intravitreal anakinra inhibits choroidal neovascular membrane growth in a rat model. Ocul Immunol Inflamm. 2009; 17:195-200.

40 Relton JK, Rothwell NJ. Interleukin-1 receptor antagonist inhibits ischaemic and excitotoxic neuronal damage in the rat. Brain Res Bull. 1992;29:243-6.

41 Hanyuda N, Akiyama H, Shimoda Y, Mukai $\mathrm{R}$, Sano M, Shinohara $Y$, et al. Different filling patterns of the choriocapillaris in fluorescein and indocyanine green angiography in primate eyes under elevated intraocular pressure. Invest Ophthalmol Vis Sci. 2017;58: 5856-61.

42 Takahashi K, Muraoka K, Kishi S, Shimizu K. Watershed zone in the human peripheral choroid. Ophthalmology. 1996;103:336-42.

43 Lepore D, Quinn GE, Molle F, Orazi L, Baldascina A, Ji MH, et al. Follow-up to age 4 years of treatment of type 1 retinopathy of prematurity intravitreal bevacizumab injection versus laser: fluorescein angiographic findings. Ophthalmology. 2018; 125:218-26. 